\title{
Rituximab therapy in steroid resistant thyroid associated ophthalmopathy
}

\author{
Praveen Devarbhavi, Animesh Maiti, Anirban Sinha, Asish Kumar Basu, \\ Satyam Chakraborty, Lakshmi Dey, Subir Swar, Kingshuk Bhattacharjee
}

\begin{abstract}
Introduction:

Thyroid

associated ophthalmopathy (TAO) is the most frequent extrathyroidal manifestation of Graves' disease. Considerable number of TAO is also found with euthyroid or hypothyroid state and responds to present mainstay of therapy i.e corticosteroid. However, some patients with TAO are resistant to steroid therapy and may progress to vision threatening orbitopathy. Radiotherapy, surgical decompression and cyclosporine in combination with oral or intravenous glucocorticoid were the other options in such cases. But recently rituximab a monoclonal anti CD2o antibody which was successfully used in other
\end{abstract}

Praveen Devarbhavi ${ }^{1}$, Animesh Maiti ${ }^{2}$, Anirban Sinha ${ }^{3}$, Asish Kumar Basu' ${ }^{4}$, Satyam Chakraborty ${ }^{5}$, Lakshmi Dey ${ }^{5}$, Subir Swar $^{5}$, Kingshuk Bhattacharjee ${ }^{6}$

Affiliations: ${ }^{1} \mathrm{DM}$ Post-Doctoral Trainee, Department of Endocrinology and Metabolism, Medical College and Hospital, 88, College Street; Kolkata; ${ }^{2}$ Associate Professor, Department of Endocrinology and Metabolism, Medical College and Hospital, 88, College Street; Kolkata; ${ }^{3}$ Assistant professor, Department of Endocrinology and Metabolism, Medical College and Hospital, 88, College Street; Kolkata; ${ }^{4}$ Head of Department, Department of Endocrinology and Metabolism, Medical College and Hospital, 88, College Street; Kolkata; ${ }^{5}$ DM Post-Doctoral Trainee, Department of Endocrinology and Metabolism, Medical College and Hospital, 88, College Street; Kolkata; ${ }^{6}$ Assistant Manager, Medical Services, Biocon LTD, Bangalore.

Corresponding Author: Dr. Praveen Devarbhavi, DM PostDoctoral Trainee, Department of Endocrinology and Metabolism, Medical College and Hospital, 88, College Street; Kolkata 700073

Received: 12 December 2016 Accepted: 24 February 2017 Published: 08 April 2017 autoimmune diseases like rheumatoid arthritis is now introduced as a novel therapeutic option in TAO particularly in steroid resistant cases. Herein, we report a case of euthyroid TAO resistant to glucocorticoid therapy that responded to rituximab.

Keywords: Rituximab, Steroid resistant orbitopathy, Thyroid associated ophthalmopathy

\section{How to cite this article}

Devarbhavi P, Maiti A, Singha A, Basu AK, Chakraborty S, Dey L, Swar S, Bhattacharjee K. Rituximab therapy in steroid resistant thyroid associated ophthalmopathy. Case Rep Int 2017;6:1720.

Article ID: 100036CRINTPD2017

$* * * * * * * *$

doi:10.5348/crint-2017-36-CR-5

\section{INTRODUCTION}

Thyroid associated ophthalmopathy (TAO) or frequently called Graves' orbitopathy (GO) commonly occurs in association with Graves' hyperthyroidism. The prevalence of GO in euthyroid and primarily hypothyroid patients ranges between 1.6\% and 8.6\% [1]. Orbitopathy in hypothyroid/euthyroid patients present with less soft tissue involvement, more asymmetrical disease and clinically less marked symptoms than in primarily hyperthyroid patients [1]. Determination of thyroidspecific antibodies contributes to the diagnosis of underlying thyroid disease in such situations.

The pathophysiology of TAO is still incompletely understood, but involves both humoral and cell mediated 
immune response. Orbital fibroblasts are the target cells in Graves' orbitopathy and autoantibodies diverted against thyroid stimulating hormone $\mathrm{TSH}$ receptor (TSHR) stimulates TSHR present in the orbital fibroblasts and leads to secretion of large quantity of glycosaminoglycan (hyaluronan). Sub group of these fibroblast differentiate into mature adipocyte with increased expression of TSHR on their surface and thus causing enlargement of extraocular muscle and expansion of orbital fat. This orbital fat is prone to robust inflammatory reaction within the orbit. Evidences suggest that there is an upregulation of immediate early adipogenic genes and other adipocyte-related genes, including those encoding peroxisome proliferator activated receptor (PPAR- $\gamma$ ), interleukin-6, adiponectin, and leptin. PPAR- $\gamma$ agonists stimulate adipogenesis and expression of the thyrotropin receptor in cultured orbital preadipocytes. [2]. Insulin like growth factor type 1 receptor (IGF1R) is another important autoantigen implicated in Graves orbitopathy expressed by fibroblast and serum of these patients found to have antibodies directed against these receptors [3]. Extraocular muscles, connective tissues and fat are infiltrated with lymphocytes leading to acute inflammatory changes and later scarring.

Current treatment option for moderate to severe active TAO is oral or intravenous glucocorticoid (GC), orbital radiation, surgical decompression or combination of these. Intravenous pulse methyl prednisolone therapy is preferred because of its higher efficacy and lower side effect [4]. Failure to glucocorticoid therapy is not infrequent and should be considered when decrease in the clinical activity score (CAS) of $<2$, increase in CAS $\geq$ 2 following the taper of glucocorticoid or development of dysthyroid optic neuropathy (DON) during the course of glucocorticoid therapy. RTX appears to be an attractive option in these situations as B cell depletion might be an effective strategy for salvaging these individuals by non-surgical means. Herein we report a case of moderate to severe progressive euthyroid TAO refractory to glucocorticoid therapy who responded to this biological agent.

\section{CASE REPORT}

A 46-year-old Indian male non-smoker presented in September 2014 with history of protrusion of eye ball, swelling and redness of eye for four months duration. He visited local ophthalmologist who prescribed eye drops and oral prednisolone. Patient discontinued medication after three weeks as symptoms improved and did not consult doctor again. After two months his symptoms reappeared and progressed. So he consulted ophthalmology department of our institute and was referred to us for evaluation of thyroid associated ophthalmopathy. On admission he had bilateral proptosis, swelling and redness of eyelid, conjunctival redness with chemosis and inflamed caruncle. Clinical activity score
(CAS) was $5 / 7$ bilateral and there was no diplopia. Patient was clinically euthyroid and there was no goitre. He was biochemically euthyroid (Free T4:1.3 ng/dl , TSH: 2.021 $\mathrm{mIU} / \mathrm{ml}$ ) with elevated TSHR Ab (70.6 IU/ml, Positive if $>14 \mathrm{IU} / \mathrm{ml}$ ) and elevated anti TPO antibody levels (256 U/ml, Normal <35U/ml). He received weekly pulse therapy for 12 cycles ( $500 \mathrm{mg}$ of intravenous pulse methyl prednisolone weekly for initial six cycles followed by 250 mg weekly for subsequent six cycles).There was only a mild symptomatic improvement after initial three cycles but again continued to have symptoms till at the end of therapy when CAS remained 5/7 bilaterally. The patient was lost for follow up for three month during this period he was using only local eye protective measures and presented again with worsening of eye symptoms with pain on eye ball movements with increased eyelid swelling. On examination CAS 6/7 bilateral (Figure 1A-B) NOSPECS (a standard mnemonic used for classification for thyroid associated ophthalmopathy) was $2 \mathrm{c} 3 \mathrm{~b} 4 \mathrm{a}$ with restriction in abduction of left eye. Subjective diplopia score was 2. Vision in both eyes was 6/9, with normal intra ocular pressure and fundus. Systemic examination was normal. Thyroid profile was within normal limit. Liver enzymes, metabolic and hematologic profiles were normal.

As patient did not respond to previous glucocorticoid therapy other options like radiotherapy, surgical decompression and biological agent rituximab were discussed. As patient was unwilling for either radiotherapy or surgical option RTX was planned after detailed discussion about pros and cons of therapy. He was screened for chronic infection like tuberculosis hepatitis B, C and HIV. Premedications included $125 \mathrm{mg}$ intravenous methylprednisolone, $1 \mathrm{~g}$ oral acetaminophen, and $50 \mathrm{mg}$ diphenhydramine, given 30 minutes prior to RTX infusion. Patient was given $500 \mathrm{mg}$ of RTX through intravenous infusion. Infusion was repeated after two weeks. He did not develop any major adverse reaction and was discharged with cotrimoxazole prophylaxis. The patient was assessed two weeks following 2nd dose and again at sixth week and third month. At sixth week CAS was $3 / 7$, and at third month CAS was $2 / 7$ (Figure 2A-B) NOSPECS was 2a3a, with improvement in proptosis. There was no diplopia with normal range of ocular movement without any deterioration of vision. After 12 months of last therapy with RTX, the patient became hypothyroid and was replaced with thyroxine and clinical activity score was stabilized to $2 / 7$.

\section{DISCUSSION}

Thyroid-associated ophthalmopathy (TAO), affecting 30-50\% of patients with Graves' disease, can be found with euthyroid and hypothyroid subjects. They usually respond to steroid therapy when compared to TAO associated with hyperthyroidism. Up to $20 \%$ of patients of active Graves' orbitopathy are unresponsive to present intravenous glucocorticoid therapy and 
another 10-20\% relapses after steroid withdrawal and up to $5 \%$ may progress to dysthyroid optic neuropathy even on treatment $[5,6]$. The patient had modrate to severe active TAO and did not respond to intravenous glucocorticoid therapy. This is unusual in euthyroid TAO who develop only milder disease and respond promptly to glucocorticoid therapy.

Thyroid associated ophthalmopathy results from B cells driven mechanism leading to production of TSHR antibody, which acts through thyrotropin receptors present on orbital fibroblasts leading to production of hyaluronan and also differentiation in to adipocytes. These B cells are effective antigen presenting cells leading to activation of $\mathrm{T}$ cells and also involved in production of proinflammatory cytokines $[2,6]$. Since RTX is a human/ murine chimeric monoclonal antibody which targets CD20, transmembrane protein present on immature and mature B cells, effectively depletes these cells from periphery leading to reduction of pathogenic TSHR antibodies and inflammation without affecting generation of B cells from bone marrow as CD 20 is absent on most pro-B cell or plasma cells [6]. Additionally, RTX reduces production of autoantibody to IGF-1R which are involved in pathogenesis of a subset of TAO [7]. Apart from these RTX may also lead to clinical benefit through altered levels of regulatory $\mathrm{T}$ cells (Tregs) which are involved in maintaining tolerance to self-antigens [8].

As natural course of TAO has acute inflammation for 6-12 months followed by stabilization of clinical signs, a transient (4-6 months) B cell depletion induced by RTX is potential therapeutic strategy. Our patient responded to RTX therapy with significant reduction in disease activity.

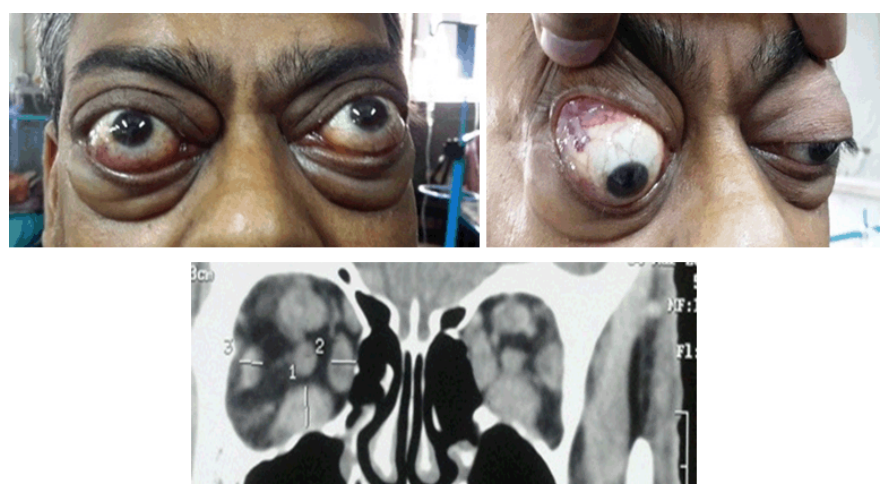

Figure 1: (A) Eye signs before rituximab therapy. CAS 6/7, with proptosis, (B) Dacryoadenitis with congestion, (C) Coronal computed tomography scan of orbit showing symmetric enlargement of extraocular muscles of both the orbit.

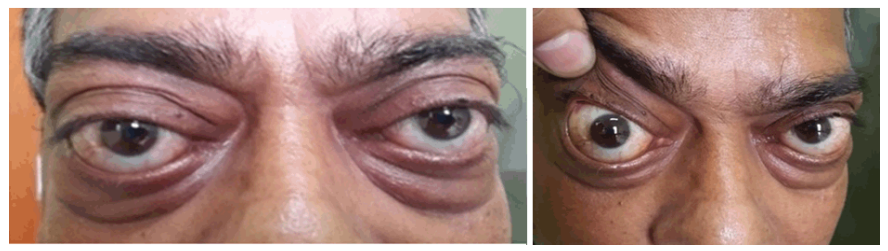

Figure 2: (A) Eye signs improved at 12th week following rituximab therapy, (B) Lacrimal gland swelling disappeared with improvement in soft tissue swelling and congestion.
Initial studies by Fassi et al. and Salvi et al. has shown that blocking the CD2O receptor on B-lymphocytes with rituximab affects the clinical course of TAO, by reducing inflammation and the degree of proptosis [9-11] Khanna et al. have treated six patients with active and severe Gravis' orbitopathy, unresponsive to glucocorticoids with RTX, and have observed a decrement in the CAS from 5.5-1.8 at eight week, which remained low at sixth month [12]. In all these studies, there was no relapse in patient treated with RTX. So far only two randomized control trials are published. Stan et al. from Mayo clinic while studying efficacy of rituximab in 25 Graves' orbitopathy (RTX $(n=13)$ vs Placebo $(n=12))$ found that RTX offered no additional benefit over placebo. They also found that duration of the disease was important determinant [5]. Duration more than one year may not respond to RTX therapy. In contrast Mario Salvi et al. showed better therapeutic outcome of RTX in active moderate to severe Graves' orbitopathy, when compared to intravenous methylprednisolone. Findings from the two studies were discrepant and more RCT's need to be done to establish the role of RTX in treatment of steroid resistant Graves' orbitopathy.

Most frequently reported side effects are Infusionrelated reactions. It can occur with mild febrile illness to hypotension. Rarely urinary tract infection, worsening of hypertension, progressive multifocal leukoencephalopathy, opportunistic infections and sudden cardiac death can occur. Our patient did not have any of the side effects related to RTX.

\section{CONCLUSION}

This case highlights the use of rituximab as a potential therapeutic option in steroid resistant active moderate to severe thyroid associated ophthalmopathy (TAO). Two RCTs done to evaluate efficacy of RTX in Graves' orbitopathy have shown contrasting results. Limited by small numbers these studies call for larger multicentric trials. Due to high cost and potential side effects of rituximab therapy, it should be restricted to severe steroid resistant thyroid associated orbitopathy. Looking at the encouraging results further studies are needed to see efficacy of low dose RTX in such cases which indeed may reduce cost and side effects significantly.

$$
* * * * * * * * *
$$

\section{Author Contributions}

Praveen Devarbhavi - Substantial contributions to conception and design, Acquisition of data, Analysis and interpretation of data, Drafting the article, Revising it critically for important intellectual content, Final approval of the version to be published

Animesh Maiti - Analysis and interpretation of data, Revising it critically for important intellectual content, Final approval of the version to be published 
Anirban Sinha - Analysis and interpretation of data, Revising it critically for important intellectual content, Final approval of the version to be published

Asish Kumar Basu - Analysis and interpretation of data, Revising it critically for important intellectual content, Final approval of the version to be published

Satyam Chakraborty - Analysis and interpretation of data, Revising it critically for important intellectual content, Final approval of the version to be published Lakshmi Dey - Analysis and interpretation of data, Revising it critically for important intellectual content, Final approval of the version to be published

Subir Swar - Analysis and interpretation of data, Revising it critically for important intellectual content, Final approval of the version to be published

Kingshuk Bhattacharjee - Analysis and interpretation of data, Revising it critically for important intellectual content, Final approval of the version to be published

\section{Guarantor}

The corresponding author is the guarantor of submission.

\section{Conflict of Interest}

Authors declare no conflict of interest.

\section{Copyright}

(C) 2017 Praveen Devarbhavi et al. This article is distributed under the terms of Creative Commons Attribution License which permits unrestricted use, distribution and reproduction in any medium provided the original author(s) and original publisher are properly credited. Please see the copyright policy on the journal website for more information.

\section{REFERENCES}

1. Eckstein AK, Lösch C, Glowacka D, et al. Euthyroid and primarily hypothyroid patients develop milder and significantly more asymmetrical Graves ophthalmopathy. Br J Ophthalmol 2009 Aug;93(8):1052-6.
2. Bahn RS. Graves' ophthalmopathy. N Engl J Med 2010 Feb 25;362(8):726-38.

3. Smith TJ. The putative role of fibroblasts in the pathogenesis of Graves' disease: Evidence for the involvement of the insulin-like growth factor-1 receptor in fibroblast activation. Autoimmunity 2003 Sep-Nov;36(6-7):409-15.

4. Bartalena L, Baldeschi L, Dickinson A, et al. Consensus statement of the European Group on Graves' orbitopathy (EUGOGO) on management of GO. Eur J Endocrinol 2008 Mar;158(3):273-85.

5. Stan MN, Garrity JA, Carranza Leon BG, et al. Randomized controlled trial of rituximab in patients with Graves' orbitopathy. J Clin Endocrinol Metab 2015 Feb;100(2):432-41.

6. Salvi M, Vannucchi G, Beck-Peccoz P. Potential utility of rituximab for Graves' orbitopathy. J Clin Endocrinol Metab 2013 Nov;98(11):4291-9.

7. AMcCoy AN, Kim DS, Gillespie EF, Atkins SJ, Smith TJ, Douglas RS. Rituximab (Rituxan) therapy for severe thyroid-associated ophthalmopathy diminishes IGF-1R(+) T cells. J Clin Endocrinol Metab 2014 Jul;99(7):E1294-9.

8. Boissier MC, Assier E, Biton J, Denys A, Falgarone G, Bessis N. Regulatory T cells (Treg) in rheumatoid arthritis. Joint Bone Spine 2009 Jan;76(1):10-4.

9. El Fassi D, Nielsen CH, Hasselbalch HC, Hegedüs L. Treatment-resistant severe, active Graves' ophthalmopathy successfully treated with B lymphocyte depletion. Thyroid 2006 Jul;16(7):70910.

10. Salvi M, Vannucchi G, Campi I, et al. Treatment of Graves' disease and associated ophthalmopathy with the anti-CD20 monoclonal antibody rituximab: An open study. Eur J Endocrinol 2007 Jan;156(1):33-40.

11. Salvi M, Vannucchi G, Currò N, et al. Efficacy of B-cell targeted therapy with rituximab in patients with active moderate to severe Graves' orbitopathy: A randomized controlled study. J Clin Endocrinol Metab 2015 Feb;100(2):422-31.

12. Khanna D, Chong KK, Afifiyan NF, et al. Rituximab treatment of patients with severe, corticosteroidresistant thyroid-associated ophthalmopathy. Ophthalmology 2010 Jan;117(1):133-139.e2.
Access full text article on other devices

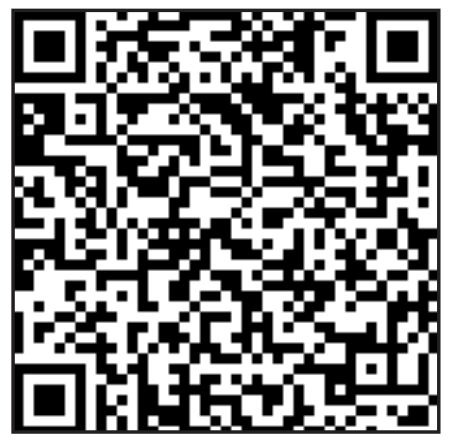

Access PDF of article on other devices

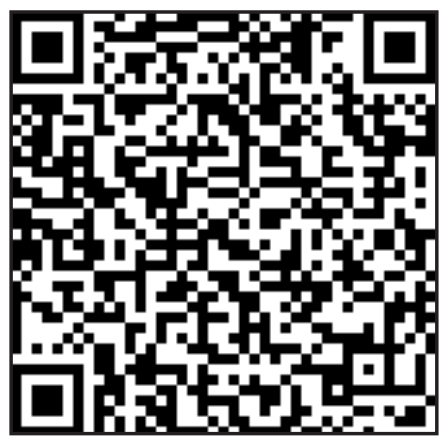

